\title{
For obese postmenopausal women, what options may decrease endometrial cancer risk?
}

\author{
Intentional weight loss, including diet, exercise, and bariatric surgery, as well \\ as progestin treatment, may help this population of women reduce their risk \\ of endometrial cancer
}

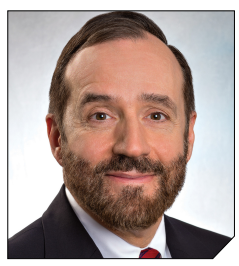

\author{
Robert L. Barbieri, MD \\ Chair Emeritus, Department of Obstetrics and Gynecology \\ Interim Chief, Obstetrics \\ Brigham and Women's Hospital \\ Kate Macy Ladd Distinguished Professor of Obstetrics, \\ Gynecology and Reproductive Biology \\ Harvard Medical School \\ Boston, Massachusetts
}

ra ndometrial cancer is the most common gynecologic malignancy, with approximately 59,000 cases diagnosed annually, ${ }^{1}$ and a lifetime risk of approximately $3.1 \%$ in the United States. ${ }^{2}$ Type I endometrial cancer includes tumors with endometrioid histology that are grade 1 or 2 . Type II endometrial cancer includes tumors that have grade 3 endometrioid or nonendometrioid histology, including serous, clear cell, mucinous, squamous transitional cell, mesonephric, and undifferentiated tumors. ${ }^{3}$ Type I endometrial cancer is hormone sensitive, generally stimulated by estrogen and suppressed by progestins.

Endometrial cancer is diagnosed at a mean age of 63 years, ${ }^{4}$ and only $15 \%$ of cases occur before age $50 .{ }^{5}$ Women with an elevated body mass index (BMI) have a markedly increased risk of both Types I and II endometrial cancer (TABLE, page 10). ${ }^{6}$ Hence, endometrial cancer is highly

doi: 10.12788/obgm.0055 prevalent in obese postmenopausal women. For these women health interventions that may reduce the risk of developing endometrial cancer include dieting, physical activity, bariatric surgery, and progestin therapy.

\section{Educating patients is a priority} Many women do not know that postmenopausal bleeding is a sign of endometrial cancer. All postmenopausal women should be advised that if they develop vaginal bleeding they need to be evaluated by a clinician. ${ }^{7}$ Women who are knowledgeable about the link between postmenopausal vaginal bleeding and endometrial cancer can be encouraged to share this information with their postmenopausal friends in order to reach more people with this important information. All obese postmenopausal women should be advised that weight loss and increased physical activity can reduce the risk of developing endometrial cancer.

\section{How weight loss and physical} activity affect risk

Intentional weight loss has been reported to reduce the risk of endometrial cancer in postmenopausal women. As part of the Women's Health Initiative observational study, 36,794 postmenopausal women aged 50 to 79 years with a uterus had their body weight and height measured at entry into the study and after 3 years of follow-up. ${ }^{8}$ During the 11 years following study entry, there were 566 incident cases of endometrial cancer. Compared with women who had a stable weight, intentional weight loss of $\geq 5 \%$ was associated with a $40 \%$ reduction in the risk of endometrial cancer (hazard ratio [HR], 0.60; 95\% confidence interval [CI], 0.42-0.86). Compared with women who had a stable weight, women who had weight gain $\geq 10 \%$ had an increased risk of endometrial cancer (HR, 1.26; 95\% CI, 1.00-1.57).

High levels of physical activity may be associated with a decreased risk of endometrial cancer. In one 
TABLE Association of BMI and risk for Type I and Type II endometrial cancer ${ }^{6}$

\begin{tabular}{c|c|c}
\hline BMI, $\mathbf{k g} / \mathbf{m}^{2}$ & Type I endometrial cancer (OR) & Type II endometrial cancer (OR) \\
\hline$<25$ & 1.00 & 1.00 \\
\hline 25 to $<30$ & 1.45 & 1.16 \\
\hline 30 to $<35$ & 2.52 & 1.73 \\
\hline 35 to $<40$ & 4.45 & 2.15 \\
\hline$>40$ & 7.14 & 3.11 \\
\hline Abbreviations: BMl, body mass index; OR, odds ratio.
\end{tabular}

study, compared with a sedentary lifestyle, higher levels of physical activity were reported to be associated with a decreased risk of endometrial cancer. ${ }^{9}$

\section{How bariatric surgery affects risk}

Many cancers are associated with obesity, including endometrial, breast, colon, pancreas, gallbladder, and renal. Obesity is associated with increased conversion of androgens to estrogens in fat tissue, stimulating excessive endometrial proliferation and increasing the risk of endometrial hyperplasia and cancer. Bariatric surgery reliably causes sustained weight reduction. Multiple studies have reported that bariatric surgery reduces the risk of endometrial cancer.

Schauer and colleagues used data from the Kaiser Permanente health system to identify 22,198 obese people who had undergone bariatric surgery and 66,427 matched controls who were obese but did not have surgery. ${ }^{10}$ The study population was $81 \%$ female, with a mean age of 45 years and a mean BMI of $45 \mathrm{~kg} / \mathrm{m}^{2}$. After an average 3.5 years of followup there were 2,542 incident cases of cancer, including 322 cases of endometrial cancer. Compared with conventional weight loss treatment, bariatric surgery reduced the risk of endometrial cancer by $50 \%$ (HR, 0.50; 95\% CI, 0.37-0.67; $P<.001) .{ }^{10}$ In addition, bariatric surgery reduced the risk of colon and pancreatic cancer by $41 \%$ and $54 \%$, respectively. ${ }^{10}$

In the Swedish Obese Subjects (SOS) study, 1,420 women who underwent bariatric surgery and 1,447 matched controls who received conventional obesity treatment were followed for 18 years. ${ }^{11}$ At study entry, the mean age of the women was approximately 48 years, and the mean BMI was approximately 42 $\mathrm{kg} / \mathrm{m}^{2}$. In follow-up there were 76 incident cases of endometrial cancer. Compared with women receiving conventional obesity treatment, women who had bariatric surgery had a non-statistically significant $49 \%$ decrease in the risk of developing endometrial cancer (HR, 0.51; 95\% CI, 0.24-1.10)

In a systematic review of 5 additional studies (not including publications 10 or 11) of the impact of bariatric surgery on the risk of developing endometrial cancer, the surgery was associated with a $68 \%$ risk reduction (odds ratio [OR], 0.32; 95\% CI, 0.16-0.63) compared with matched obese women that did not have surgery. ${ }^{12}$

Although there are no randomized prospective studies showing that bariatric surgery reduces the risk of endometrial cancer, the weight of the observation evidence is strong. In addition, bariatric surgery was reported to reduce all-cause mortality in the SOS study. ${ }^{13}$ Hence, for obese postmenopausal women, if lifestyle changes do not result in sustained weight loss, bariatric surgery may be an optimal approach to improving health outcomes.

\section{Progestin treatment and endometrial cancer risk}

Estrogen stimulates endometrial cell proliferation. Hence, unopposed chronic exposure to estrogen is a major risk factor for developing endometrial hyperplasia and cancer. Progestins block the proliferative effect of estrogen and cause cell differentiation, resulting in stromal decidualization. Progestins also reduce the concentration of estrogen and progesterone receptors and increase the activity of enzymes that convert estradiol to estrone, blocking estrogen-induced endometrial proliferation. ${ }^{14}$

In women with endometrial hyperplasia, progestins have been shown to be effective in resolving the hyperplasia in approximately $80 \%$ of cases. Both oral progestins and the 52-mg levonorgestrel-containing intrauterine device (LNG-IUD) have been reported to be effective in the treatment of endometrial hyperplasia. In a Cochrane systematic review 
and meta-analysis, the 52-mg LNGIUD was reported to be somewhat more effective in resolving endometrial hyperplasia than cyclic oral progestins ( $89 \%$ vs $72 \%$, respectively). ${ }^{15}$

Other studies have also reported that the $52 \mathrm{mg}$ LNG-IUD was more effective than oral progestin therapy for women with complex atypical endometrial hyperplasia. ${ }^{16}$ There are no large randomized clinical trials of progestin therapy on prevention for future development of endometrial cancer in obese postmenopausal women who have a normal endometrial histology. However, for an obese perimenopausal woman, insertion of a 52-mg LNG-IUD may help to minimize excessive uterine bleeding during the menopause transition and reduce the risk of developing endometrial hyperplasia during the early postmenopause.

\section{We can help our patients reduce their risk of endometrial cancer} Obese postmenopausal women are at increased risk for developing endometrial cancer. Gynecologists play an important role in the prevention and early detection of endometrial cancer. We can make a difference and improve the health of our obese peri- and postmenopausal women by recommending interventions that reduce the risk of endometrial cancer, thereby improving the health of our patients.

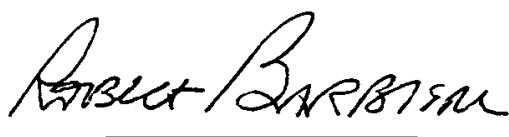

RBARBIERI@MDEDGE.COM

Dr. Barbieri reports no financial relationships relevant to this article.
References

1. American Society of Clinical Oncology. Uterine cancer statistics. https://www.cancer.net/cancer -types/uterine-cancer/statistics\#: :text=This\%20 year $\% 2 \mathrm{C} \% 20$ an $\% 20$ es timated $\% 20$ $65 \% 2 \mathrm{C} 620$,cancers\%20occur\%20in\%20the $\% 20$ endometrium. Accessed November 23, 2020.

2. Howlader N, Noone AM, Krapcho M, et al (eds). SEER Cancer Statistics Review, 1975-2017. National Cancer Institute: Bethesda, MD. April 15, 2020. https://seer.cancer.gov/csr/1975 2017/. Accessed November 23, 2020.

3. Noer MC, Antonsen SL, Ottesen B, et al. Type I versus Type II endometrial cancer: differential impact of comorbidity. Int J Gynecol Cancer. 2018;28:586-593. Sorosky JI. Endometrial cancer. Obstet Gynecol. 2008;111:436-437.

4. Gallup DG, Stock RJ. Adenocarcinoma of the endometrium in women 40 years of age or younger. Obstet Gynecol. 1984;64:417-420.

5. Setiawan VW, Yang HP, Pike MC, et al. Type I and II endometrial cancers: have they different risk factors. J Clin Oncol. 2013;31:2607-2618.

6. Saccardi C, Vitagliano A, Marchetti M, et al. Endometrial cancer risk prediction according to indication of diagnostic hysteroscopy in postmenopausa women. Diagnostics (Basel). 2020;10:257.el-ell.

7. Luo J, Chlebowski RT, Hendryx M, et al. Intentional weight loss and endometrial cancer risk. JClin Oncology. 2017;35:1189-1193.

8. Friedenreich CM, Ryder-Burbidge C, McNeil J. Physical activity, obesity and sedentary behavior in cancer etiology: epidemiologic evidence and biological mechanisms. Mol Oncol. August 2, 2020. doi: 10.1001/1878-0261.12772.

9. Schauer DP, Feigelson HS, Koebnick C, et al. Bariatric surgery and the risk of cancer in a large multisite cohort. Ann Surg. 2019;269:95-101.

10. Anvenden A, Taube M, Peltonen M, et al. Longterm incidence of female-specific cancer after bariatric surgery or usual care in the Swedish Obese Subjects Study. Gynecol Oncol. 2017;145:224-229. 11. Winder AA, Kularatna M, MacCormick AD. Does bariatric surgery affect the incidence of endometrial cancer development? A systematic review. Obes Surg. 2018;28:1433-1440.

12. Carlsson LM, Sjoholm K, Jacobson P, et al. Life expectancy after bariatric surgery in the Swedish Obese Subjects Study. $N$ Engl J Med. 2020;383:1535-1543.

13. Lessey BA, Young SL. In: Strauss JF, Barbieri RL (eds.) Yen and Jaffe's Reproductive Endocrinology: Physiology, Pathophysiology and Clinical Management. 8th ed. Elsevier Saunders: Philadelphia, PA; 2018:208-212.

14. Mittermeier T, Farrant C, Wise MR. Levonorgestrel-releasing intrauterine system for endometrial hyperplasia. Cochrane Database Syst Rev. 2020;CD012658.

15. Mandelbaum RS, Ciccone MA, Nusbaum DJ, et al. Progestin therapy for obese women with complex atypical hyperplasia: levonorgestrel-releasing intrauterine device vs systemic therapy. $A m J$ Obstet Gynecol. 2020;223:103.e1-e13. 